\title{
Simulation method improves accuracy for optical metrology and nanooptics design
}

\section{Sven Burger}

A dedicated finite-element solver enables accurate and fast simulations of light interaction with metallic nanostructures.

With advances in nanotechnology growing at a fast pace, reliable simulations of nanooptical devices are crucial for research and development. Designing and optimizing the functionality of nanooptical components is typically assisted by computer simulations in combination with optical inspection as a critical step in product development and quality control. Dimensional metrology, for example, relies essentially on a numerical comparison of measurements with simulations. In addition, the latter are used in most scientific nanooptics publications to support theoretical and experimental results.

The evolution of electric and magnetic fields in any welldefined nanostructure is described by Maxwell's equations. All information about the classical properties of light is contained in the electromagnetic fields, which is used to solve these differential equations. A variety of methods exist to perform rigorous simulations of Maxwell's equations. These include finite-element, finite-difference time-domain, Fouriermodal, and boundary-element approaches. However, especially
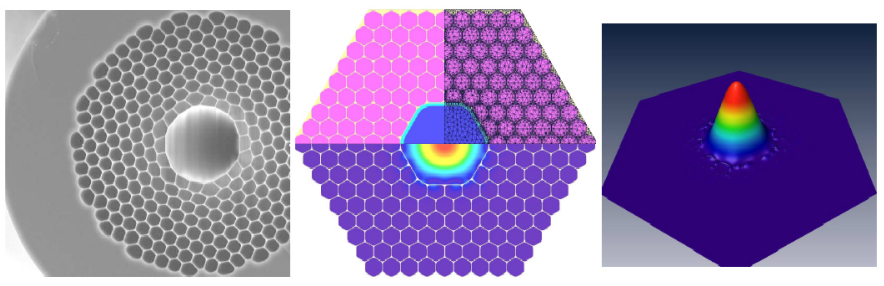

Figure 1. Efficient finite-element-method simulations for photoniccrystal fibers. (left) Microscopic image. (Courtesy B. Mangan.) (center) Collage of geometry, triangular mesh, and numerical solution. (right) Quasi-3D image of a guided mode.
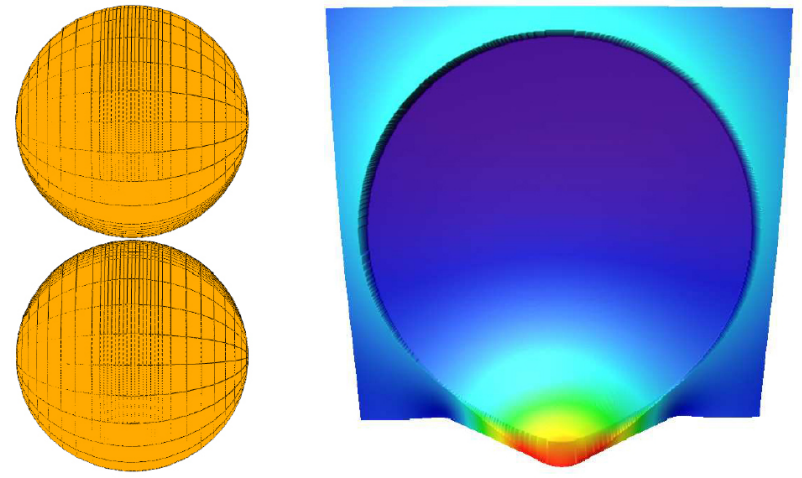

Figure 2. (left) Two gold spheres, with diameters of $80 \mathrm{~nm}$, separated by a gap of $1 \mathrm{~nm}$. Illumination at a wavelength of $632 \mathrm{~nm}$ excites a plasmon resonance. (right) Detail (section through upper sphere) of the electricfield intensity distribution (logarithmic scale, blue: low, red: high).

for large 3D computational domains and highly conductive materials (like silver or gold), the electromagnetic fields of interest are so complicated that reaching accurate approximations becomes challenging for all numerical methods. It has even been suspected that nearly all published numerical results of light scattering off 3D metal nanostructures are of incorrect accuracy. ${ }^{1}$ To approach this challenge, we use dedicated finite-element method (FEM)-based simulation tools, which enable significantly improved accuracy and relatively short computation times.

Dedicated FEM solver for nanooptics simulations In a joint effort between the Zuse Institute Berlin and JCMwave, we are developing the JCMsuite finite-element solver, a dedicated tool for nanooptical simulations. It has been applied successfully to a wide range of electromagnetic-field computations including metamaterials, ${ }^{2}$ photonic-crystal fibers ${ }^{3}$ (see Figure 1),

Continued on next page 
near-field microscopy, ${ }^{4}$ and optical microlithography. ${ }^{5}$ The performance of our methods is investigated and compared to competing approaches in a number of benchmark studies. ${ }^{5-7}$ The main ingredients for accurate performance are adaptive methods based on goal-oriented error estimators, higher-order vector elements, and fast direct and iterative numerical methods for solving matrix equations. ${ }^{8}$

\section{Field enhancement of plasmonic nanostructures}

Plasmon resonances can be used to tailor local field enhancements near metal nanostructures for specific applications. Figure 2 shows an example of two gold spheres separated by a small gap. When light of the proper wavelength $\lambda_{0}$ hits the structure, the electric-field intensity in the gap is enhanced by more than five orders of magnitude. These high local fields show promise for applications such as single-biomolecule sensing. Because of the multiscale geometry (gap $\sim 1 \mathrm{~nm}$ versus $\lambda_{0} \sim 1 \mu \mathrm{m}$ ) and the highly conductive materials, this simple 3D setup presents a challenging problem for most rigorous Maxwell solvers. Figure 2 shows a detail of the simulated field distribution at resonance. FEM allows computation of the field enhancement to a relative accuracy of approximately one percent in a computation time of $\sim 5 \mathrm{~s}$, and to an accuracy of about $0.01 \%$ in $\sim 2 \mathrm{~min}$ on a standard PC. ${ }^{7}$

Light propagation through apertures in metal films

Light interaction with structured metal films (photomasks) plays an essential role in optical lithography. Figure 3 shows the simulated intensity in a cross section through a Z-shaped nanoaperture (microlithography test case, lateral domain size approximately $10 \lambda_{0} \times 10 \lambda_{0}$, thickness $\left.\sim \lambda_{0}\right)$. ${ }^{6}$ Note the characteristic intensity discontinuities at metal-vacuum interfaces and the smooth distribution after aerial imaging. Results from a
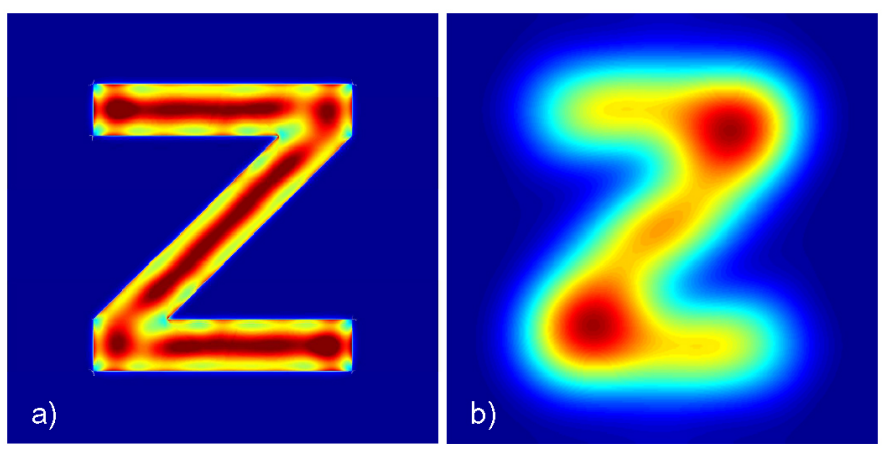

Figure 3. (a) Electric-field intensity (I) of a chromium film with a Z-shaped nanoaperture (blue: low, red: high I). (b) Aerial image (demagnified).

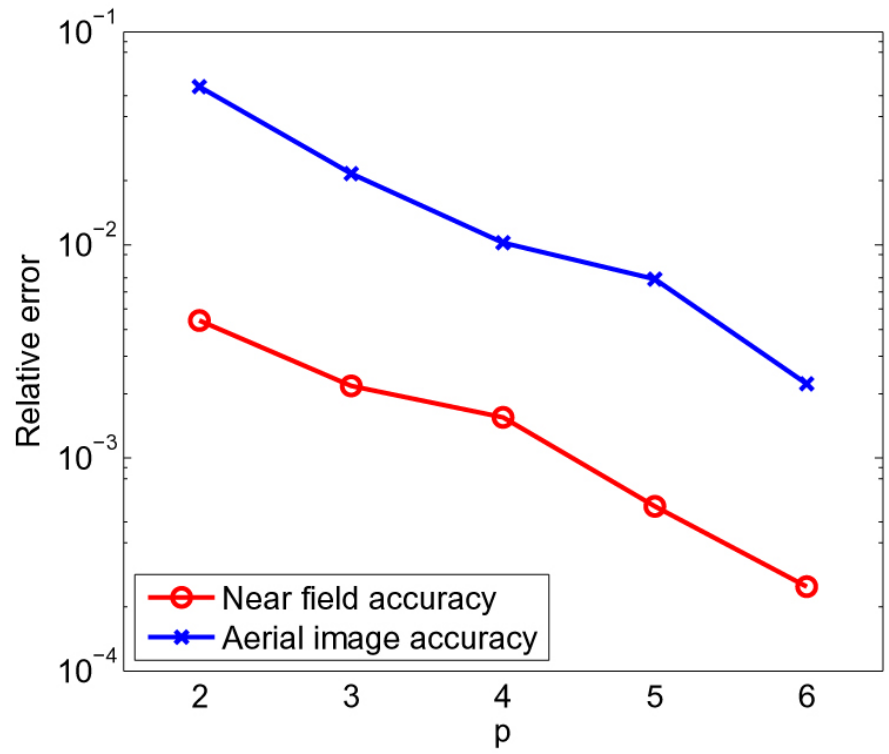

Figure 4. Convergence study for light propagation through a nanoaperture (see Figure 3). Relative errors in diffraction amplitudes (o) and aerial-image-feature widths $(\times)$ decrease with increasing discretization parameter $p$ (finite-element degree).

convergence check for this test case are displayed in Figure 4. We compare the accuracies, which are reached at specific numerical settings for the amplitude of a diffraction order, and for the width of a specific feature in the aerial image. The relative error of the observable in the aerial image is at least one order of magnitude larger than the relative near-field error. This is contrary to the widespread misunderstanding that smoothing effects in aerial imaging would 'forgive' inaccurate near-field solutions. In dedicated experimental setups, light propagation through nanoapertures in metal films can lead to unexpected optical phenomena like enhanced transmission and beaming effects. ${ }^{9}$ Numerical simulations can help to design the corresponding devices and to understand the underlying physics. ${ }^{10}$

\section{Fast simulations for optical metrology}

FEM is the method of choice for accurate and fast simulations of light interaction with nanostructures. We also use FEM for pattern reconstruction in optical metrology. ${ }^{11}$ However, optical inspection in a production environment requires very short computation times for high throughput. When complex patterns are involved, this can be an extremely demanding numerical task. We currently follow two promising approaches to overcome this challenge. First, we split simulations into 
'expensive' offline tasks that typically have long computation times and only need to be performed once, and 'cheap' online simulations with very short computation times that are done often, to enable us to generate rigorous solutions to Maxwell's equations in real-time applications. ${ }^{12}$ Secondly, parallelized domain-decomposition approaches enable accurate solutions for very large computational domains. ${ }^{13}$

\section{Author Information}

\section{Sven Burger}

Computational Nanooptics Group

Zuse Institute Berlin

Berlin, Germany

http:/ / www.zib.de/burger

http://www.zib.de/

\section{References}

1. G. Granet and L. Li, Convincingly converged results for highly conducting periodically perforated thin films with square symmetry, J. Opt. A-Pure Appl. Opt. 8, p. 546, 2006.

2. C. Enkrich, M. Wegener, S. Linden, S. Burger, L. Zschiedrich, F. Schmidt, C. Zhou,

T. Koschny, and C. M. Soukoulis, Magnetic metamaterials at telecommunication and visible frequencies, Phys. Rev. Lett. 95, p. 203901, 2005.

3. F. Couny, F. Benabid, P. J. Roberts, P. S. Light, and M. G. Raymer, Generation and photonic guidance of multi-octave optical-frequency combs, Science 318, pp. 1118-1121, 2007.

4. T. Kalkbrenner, U. Hakanson, A. Schädle, S. Burger, C. Henkel, and V. Sandoghdar, Optical microscopy using the spectral modifications of a nano-antenna Phys. Rev. Lett. 95, p. 200801, 2005.

5. S. Burger, R. Köhle, L. Zschiedrich, W. Gao, F. Schmidt, R. März, and C. Nölscher, Benchmark of FEM, waveguide and FDTD algorithms for rigorous mask simulation, Proc. SPIE 5992, p. 378, 2005. doi:10.1117/12.631696

6. S. Burger, L. Zschiedrich, F. Schmidt, P. Evanschitzky, and A. Erdmann, Benchmark of rigorous methods for electromagnetic field simulation, Proc. SPIE 7122, p. 71221S, 2008. doi: $10.1117 / 12.801248$

7. J. Hoffmann, C. Hafner, P. Leidenberger, J. Hesselbarth, and S. Burger, Comparison of electromagnetic field solvers for the $3 D$ analysis of plasmonic nano antennas, Proc. SPIE 7390. In press.

8. J. Pomplun, S. Burger, L. Zschiedrich, and F. Schmidt, Adaptive finite element method for simulation of optical nano structures, Phys. Status Solidi B 244, p. 3419, 2007.

9. H. J. Lezec, A. Degiron, E. Devaux, R. A. Linke, L. Martin-Moreno, F. J. GarciaVidal, and T. W. Ebbesen, Beaming light from a subwavelength aperture, Science 297, p. 820,2002

10. S. Burger, B. Kleemann, L. Zschiedrich, and F. Schmidt, Finite element simulations of light propagation through circular subwavelength apertures, Proc. SPIE 7366. In press. 11. F. Scholze, C. Laubis, U. Dersch, J. Pomplun, S. Burger, and F. Schmidt, The influence of line edge roughness and $C D$ uniformity on EUV scatterometry for $C D$ characterization of EUV masks, Proc. SPIE 6617, p. 66171A, 2007. doi:10.1117/12.726159

12. Z. Zhu and F. Schmidt, An efficient and robust mask model for lithography simulation, Proc. SPIE 6924, p. 69243I, 2008. doi:10.1117/12.772987

13. L. Zschiedrich, S. Burger, A. Schädle, and F. Schmidt, A rigorous finite-element domain decomposition method for electromagnetic near field simulations, Proc. SPIE 6924, p. 692450, 2008. doi:10.1117/12.771989 